\title{
Stiffness Enhancement of a Superconducting Magnetic Bearing Using Shaped YBCO Bulks
}

\author{
James G. Storey, Mathieu Szmigiel, Fergus Robinson, Stuart C. Wimbush \\ and Rodney A. Badcock, Senior Member, IEEE
}

\begin{abstract}
High-speed superconducting motors and generators stand to benefit from superconductor magnetic levitation bearings if their stiffness characteristics can be improved. Here we investigate a novel thrust bearing geometry, comprising a conical frustum shaped permanent magnet and matching superconducting toroid and puck assembly, aimed at producing high stiffness coupled with high levitation force. To this end, we have constructed a bearing test rig enabling measurements of the levitation force and stiffness of the assembly of $\mathrm{YBa}_{2} \mathrm{Cu}_{3} \mathrm{O}_{7-\delta}$ melt-textured bulks and $\mathrm{Nd}_{2} \mathrm{Fe}_{14} B$ permanent magnet at temperatures down to $47 \mathrm{~K}$. The experimental results are supported by finite element modeling that is validated against the experiment, and used to quantify the advantages of this configuration over a conventional cylindrical magnet and HTS puck arrangement. For axial displacements, the assembly produces higher and more consistent stiffness together with stronger restoring forces. For lateral displacements, the assembly produces up to double the lateral force and up to four times the stiffness. Our study also shows that the force contribution to the assembly from the small inner puck is negligible and it can therefore be eliminated from the bearing design.
\end{abstract}

Index Terms-Superconducting magnetic bearing, levitation force, toroid, conical frustum, HTS bulks.

\section{INTRODUCTION}

$\mathbf{T}$ HE aviation industry is looking increasingly towards hybrid-electric aircraft propulsion as a means of reducing carbon-dioxide emissions, and the demanding power, weight and efficiency requirements have renewed interest in multi-megawatt high-speed superconducting motors and generators[1], [2], [3], [4]. High-stiffness low-loss bearings are a key enabling subsystem for such machines. Since cooling is already available in a superconducting machine it makes sense to consider incorporating high-temperature superconductor (HTS) levitation bearings [5], [6], featuring passive stability and extremely low rotational losses. However, compared to mechanical and active magnetic bearings, HTS bearings are limited in applications by their low stiffness[6], [7].

The levitation force from truncated cone (or conical frustum) shaped permanent magnets (PM's) of varying thickness was investigated recently[8], and it was found that the force could be optimized at a particular thickness. Here, we go a step further by introducing an HTS bulk with a matching recess, into which a frustum shaped PM can settle. Our hypothesis is

This work was supported by the New Zealand Ministry of Business, Innovation and Employment (MBIE) under contract RTVU1707. (Corresponding author: James G. Storey.)

The authors are with the Robinson Research Institute, Victoria University of Wellington, Lower Hutt 5046, New Zealand (e-mail: james.storey@vuw.ac.nz).

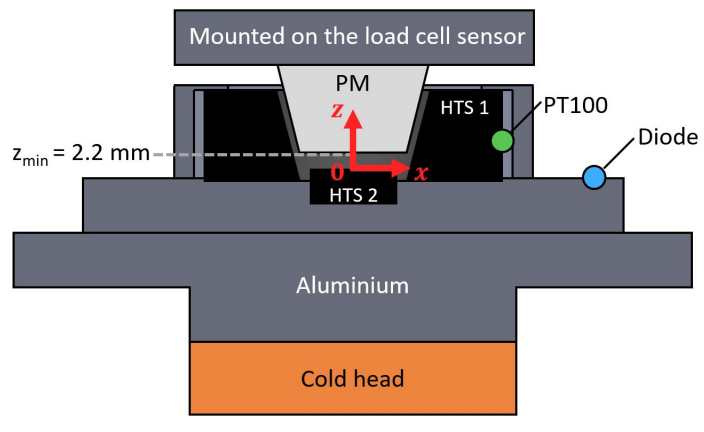

Fig. 1. Cross-sectional view of the mounting assembly of the HTS bulks to the cryocooler cold head, and of the frustum PM to the force sensor.

that this geometry provides enhanced levitation and stiffness compared to a cylindrical PM and HTS puck. In this paper we report the measured force-displacement characteristics and their analysis using $H$-formulation finite-element models.

\section{EXPERIMENTAL DETAILS}

A test rig was constructed at Robinson Research Institute to measure the vertical $(z)$ and lateral $(x$ and $y$ ) levitation forces between a conical frustum-shaped PM and an HTS assembly, composed of a trapezoidal toroid and a small puck semirecessed inside the toroid. The arrangement and dimensions of the PM and HTS are shown in Figs. 1 and 4(a). Both HTS parts are melt-textured $\mathrm{YBa}_{2} \mathrm{Cu}_{3} \mathrm{O}_{7-\delta}$ bulks supplied by Can Superconductors[9] and the N50-grade NdFeB PM was supplied by SuperMagnetMan[10].

The large face of the PM is glued to an aluminium plate which is mounted on a 3-axis load cell sensor with a range of $\pm 500 \mathrm{~N}$ in each direction. This sensor is mounted on a linear displacement system capable of lateral $(x)$ and vertical (z) movements. The displacement speed is set to $0.5 \mathrm{~mm} / \mathrm{s}$. Two linear variable differential transformer sensors provide the position of the PM. The servo motor is controlled using a TrioMotion MC464 with National Instruments CompactDAQ hardware and Labview making measurements of the analogue signals. The HTS puck is semi-recessed into an aluminium plate and secured with an aluminium filled epoxy. The HTS toroid is clamped onto the same plate with thermal contact aided by a thin layer of vacuum grease. This plate is mounted to a second aluminium plate which is attached from below to the top of the cold head of a Cryomech AL63 cryocooler. The apparatus is housed in a turbo-pumped vacuum chamber operating at a typical pressure of $10^{-5} \mathrm{hPa}$. The experimental arrangement is shown in Fig. 2. 


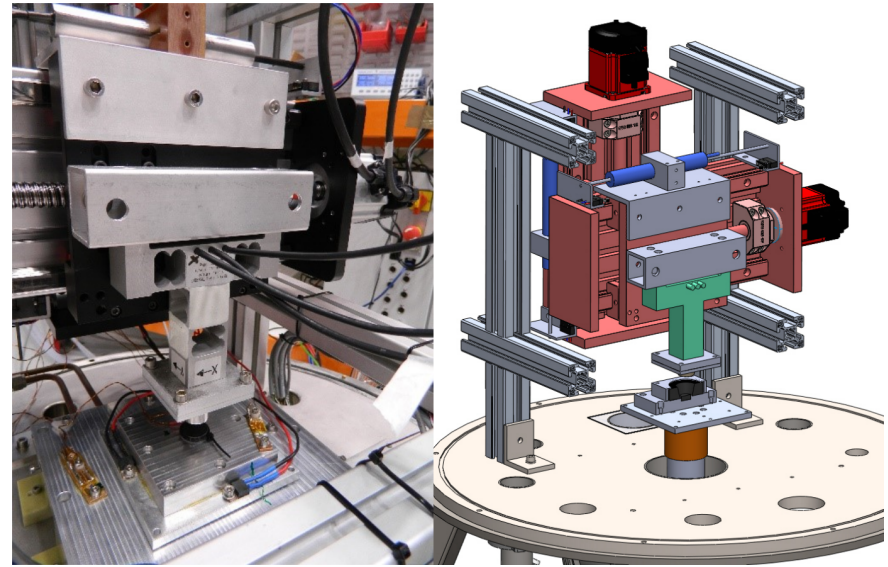

Fig. 2. Photo and drawing of the measurement test rig, showing the load cell sensor (green), displacement sensors (blue) and translational stage and motors (red). The HTS assembly and mounting plates have been sectioned for clarity.

Temperature is measured on the toroid with a PT100 resistance temperature detector attached to a flat region shaved onto the outer curved side. A diode sensor measures the temperature of the aluminium plate on which the HTS parts are mounted. The toroid temperature is maintained with a temperature controller and four heater resistors, with temperature feedback provided by the PT100. The toroid temperature can be stabilized over the range $42 \mathrm{~K}$ to $90 \mathrm{~K}$. No temperature measurement is carried out on the PM.

After moving the PM to its initial position for a field cooling (FC) or zero field cooling (ZFC) experiment the HTS assembly is cooled to the desired temperature. Next a zeroing of the load cell is performed. The $z$-position represents the distance between the top face of the small HTS puck and bottom (small) face of the PM as shown in Fig. 1. The lateral position is equal to zero when the PM and the HTS are axially aligned. Force data measured at an acquisition rate of $10 \mathrm{~Hz}$ is recorded simultaneously with the PM displacement. Once a measurement sequence at a particular temperature is completed, the HTS is warmed up above its critical temperature $\left(T_{c}\right)$ before ramping to the next temperature in order to release any trapped flux.

\section{Finite Element Simulation}

Results were compared with simulations from $H$ formulation finite element models implemented in COMSOL Multiphysics[11]. We employed the method detailed by Quéval et al.[12] wherein the field from the moving permanent magnet is applied as a time-dependent Dirichlet boundary condition on a thin air region enclosing the HTS. For a given remanent flux density $B_{r}$, the PM field $H_{e x t}(x, y, z)$ only needs to be calculated once (using an $A$-formulation finite element model) and stored in a lookup table. The N50-grade value of $B_{r}=1.4 \mathrm{~T}$ was confirmed by comparing calculations of $B_{z}$ versus $z$ above the centre of the small face of the PM with values measured with a P15A Hall sensor from Advanced Hall Sensors Ltd.[13], see Fig. 3.

The material-dependent inputs to the model come via the nonlinear resistivity. For the HTS we take a bounded power

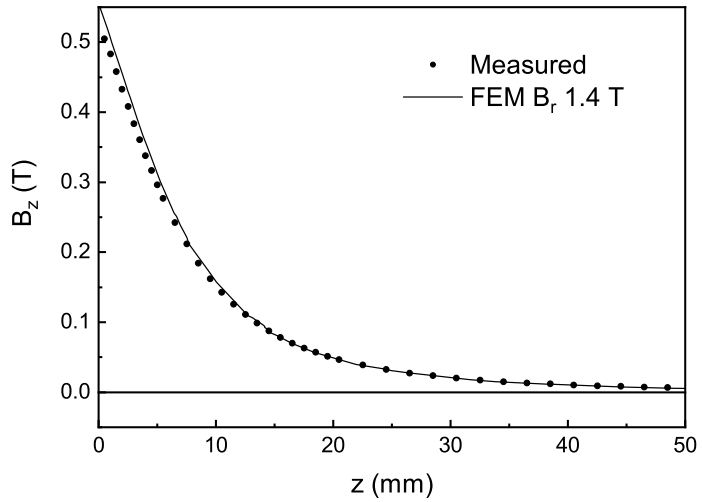

Fig. 3. Comparison of measured and modeled $B_{z}$ field as a function of the axial height, $z$, above the centre of the small face of the magnet.

law[14], [15]

$$
\rho(\mathbf{J})=\frac{\rho_{N S} \cdot \rho_{S C}(\mathbf{J})}{\rho_{N S}+\rho_{S C}(\mathbf{J})}
$$

where $\rho_{N S}=3.5 \times 10^{-6} \Omega \cdot \mathrm{m}$ is the assumed normal-state resistivity[16], and $\rho_{S C}$ is the resistivity in the superconducting state given by

$$
\rho_{S C}(\mathbf{J})=\frac{E_{c}}{J_{c}}\left|\frac{\mathbf{J}}{J_{c}}\right|^{n-1}
$$

$\mathbf{J}$ is the current density, and the $n$-value is set to 21 [17]. $J_{c}$ is the critical current density at the electric field criterion $E_{c}=10^{-4} \mathrm{~V} / \mathrm{m}$ and, in the absence of measured values, is our only adjustable parameter. For simplicity we take $J_{c}$ to be field-independent and spatially homogeneous. $J_{c}$ is assumed to be the same for both the toroid and puck. A finite resistivity of $1 \Omega \cdot \mathrm{m}$ is applied to the air region[18]. Vertical displacements of the magnet along $z$ were simulated in a $2 \mathrm{D}$-axisymmetric geometry (Fig. 4) with relative tolerance set to $10^{-4}$ and absolute tolerance set to $10^{-3}$. Mapped meshes created with an arithmetic sequence distribution method were applied to the HTS domains. A free triangular mesh was applied to the air domain. Lateral displacements along $x$ required a 3D model and the relative and absolute tolerances were relaxed to $10^{-3}$ and $10^{-2}$ respectively, as in [12]. The $3 \mathrm{D}$ mesh was created by sweeping the 2D-axisymmetric mesh around the $z$-axis. The speed of the magnet displacement was set to $1 \mathrm{~mm} / \mathrm{s}$. The force is given by the integral of $\mathbf{J} \times \mathbf{B}$ over the HTS volumes.

\section{RESULTS}

\section{A. Vertical displacement}

1) Zero-field-cooled: Zero-field-cooling sequences with $z$ going from $43 \mathrm{~mm}$ to $2.3 \mathrm{~mm}$ and back to $43 \mathrm{~mm}$ were performed at 47, 61 and $77 \mathrm{~K}$. Forces measured in each direction are shown in Fig. 5. Reducing temperature narrows the hysteresis loops in $F_{z}$, and the maximum force at low displacement saturates. The 47 and $61 \mathrm{~K}$ runs have similar maximum $F_{z}$ values of approximately $159 \mathrm{~N}$, compared to about $150 \mathrm{~N}$ at $77 \mathrm{~K}$. The loops have a rounded appearance at low displacement, reminiscent of a bell curve, which differs from the steep negative exponential decay produced by cylindrical PM's and pucks[19]. 


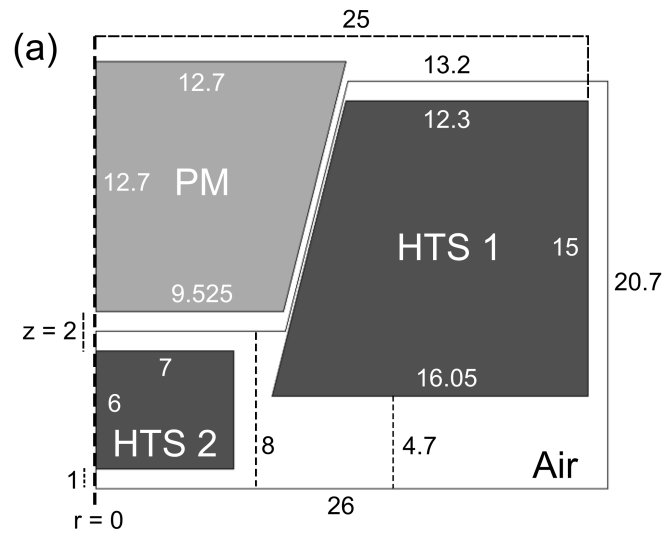

(b)

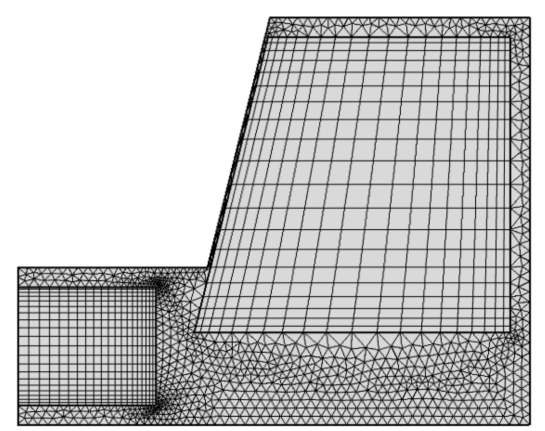

Fig. 4. (a) Axisymmetric representation of the model geometry for a vertical PM displacement of $2 \mathrm{~mm}$. HTS 1 is the toroid and HTS 2 is the puck. Dimensions are in mm. (b) $H$-formulation finite-element mesh for the axisymmetric case. The PM field is calculated separately using the $A$ formulation.

Spikes in force near the minimum displacement indicate unintentional contact between the PM and toroid. Finite forces were measured in the $x$ and $y$ directions, which should both be zero under ideal conditions. Possible explanations include an off-centre misalignment of the PM, and inhomogeneities in the HTS and/or PM[20]. The presence of spikes in $F_{y}$, together with $F_{y}$ being greater than $F_{x}$, is consistent with misalignment. But a ten percent decrease in $B_{r}$ along $y$ can also cause similar $F_{y}$ values.

Figure 5(b) shows the simulated levitation force for two values of $J_{c}, 21.6 \times 10^{8}$ and $9.6 \times 10^{8} \mathrm{~A} / \mathrm{m}^{2}$, chosen to provide a reasonable comparison with the data. Note we have not tried to perform a precise fit to the experimental data, and the same $J_{c}$ values are used in all subsequent simulations. The maximum levitation force, dictated primarily by $B_{r}$, agrees well with experiment, and the qualitative shape of the curves is reproduced. A decrease in $J_{c}$ accounts for the observed increased hysteresis and reduced force with temperature.

Contributions to the force were investigated by repeating the measurements and simulations on the puck and toroid separately. The puck produced a maximum force of $19 \mathrm{~N}$ at $67 \mathrm{~K}$ and the slope of the force curve increases monotonically as the PM approaches, see Fig. 6(a). The simulated force curve, with the same $J_{c}$, agrees very closely see Fig. 6(b). The toroid was measured at $70 \mathrm{~K}$ and the data lies between the 61 and $77 \mathrm{~K}$ data from the puck-plus-toroid assembly. If the force contributions from puck and toroid were additive we

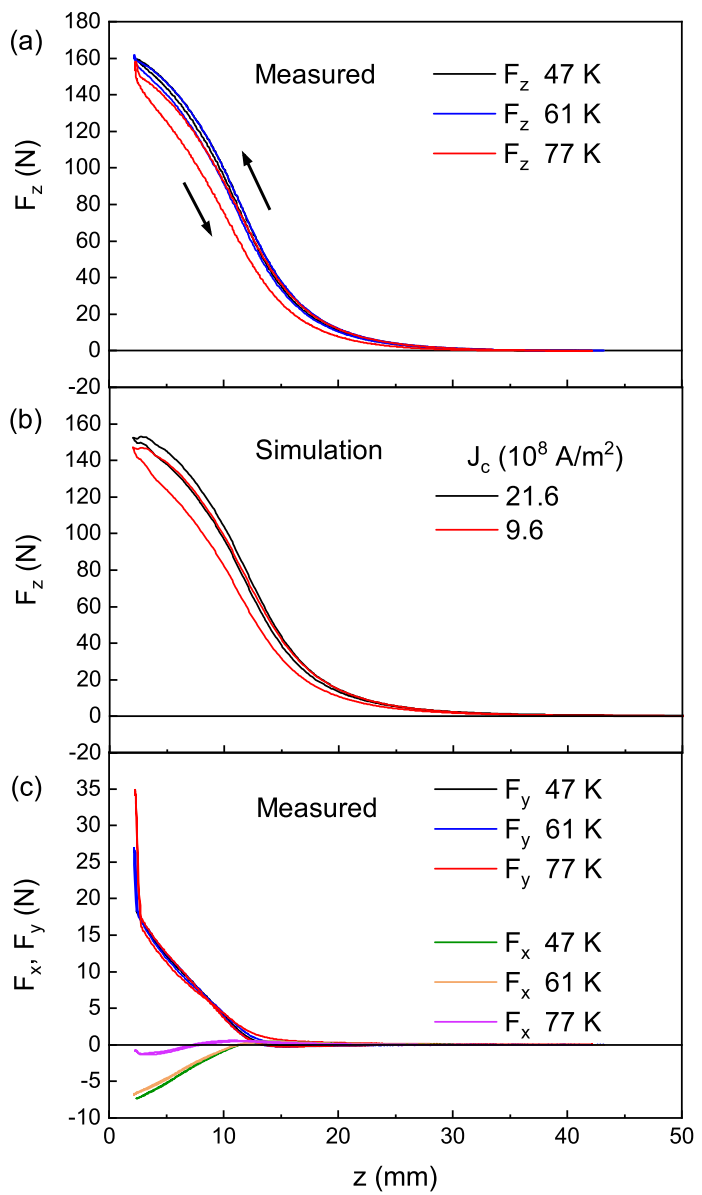

Fig. 5. Zero-field-cooled levitation force as a function of levitation height. (a) Measured $z$-component at 47,61 and 77 K. (b) Simulated $z$-component for two values of $J_{c}$. (c) Measured $x$ - and $y$-components at 47, 61 and $77 \mathrm{~K}$.

would expect the maximum of the toroid-only data to occur around $130 \mathrm{~N}$, putting it below the $77 \mathrm{~K}$ puck-plus-toroid curve. Simulation confirms that the force from the toroid is virtually the same as the assembly, suggesting that the puck is screened from the PM field by the opposing field induced in the toroid.

2) Field-cooled: Field-cooled sequences with $z$ going from 7.2 to 2.4 to 12.1 and back to $7.2 \mathrm{~mm}$ were performed at 47 and $70 \mathrm{~K}$, see Fig. 7. Maximum force at $z=2.4 \mathrm{~mm}$ is 120 $\mathrm{N}$, which is still appreciable and good from an applications perspective. The restoring force at $z=12.1 \mathrm{~mm}$ is $-52 \mathrm{~N}$. Note that the maximum force depends upon the field-cooled height of the PM, and field cooling at lower heights produces lower maximum levitation force but higher vertical stiffness. The 70 $\mathrm{K}$ data has larger hysteresis and a slight decrease in force compared to the $47 \mathrm{~K}$ data. Again, there is good qualitative and quantitative agreement between the measurements and simulations shown in panel (b). As in the ZFC case, we measured a non-zero $F_{x}$ and $F_{y}$, probably due to a slight misalignment of the PM in the $y$-direction.

\section{B. Lateral displacement}

Lateral force displacement loops, with $x$ traversing the sequence $[0,1,-1,1,-1,0] \mathrm{mm}$, were measured at $54 \mathrm{~K}$ 


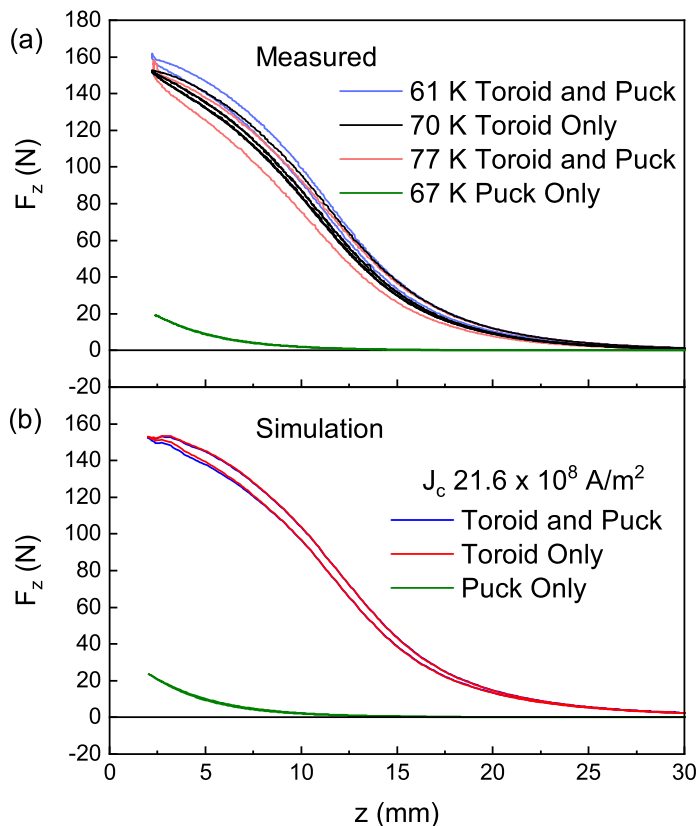

Fig. 6. Comparison of the zero-field-cooled levitation forces from the puck and toroid in isolation and in combination. Measurements in (a) and simulations in (b).

after field cooling with the PM at $z=5.2,4.2$ and $3.2 \mathrm{~mm}$, see Fig. 8. The conical geometry accommodates wider lateral motion at higher $z$. Stiffness values determined from a linear fit are $-14.7,-16.6$ and $-19.3 \mathrm{~N} / \mathrm{mm}$ respectively. There is a very slight cubic term to the elastic contribution. The loops are very narrow, indicating weak damping. The maximum $z$ force is about ten percent of the maximum $x$-force, and the maximum $y$-force is of the order of a few percent.

The simulated force curves, also in Fig. 8, do not exhibit hysteresis. $F_{x}$ is almost linear, with a small cubic component, and the stiffness increases with decreasing $z . F_{y}$ consists of discretization noise and should ideally be zero. $F_{z}$ is slightly noisy but exposes an offset in the experimental data of approximately $0.5 \mathrm{~mm}$.

\section{DISCUSSION AND CONCLUSIONS}

The agreement between measurements and simulations shows that our assumptions regarding $J_{c}$ are sound. According to the diode sensor, the recessed puck and base of the toroid could be up to $28 \mathrm{~K}$ colder than the PT100 temperature near the top of the toroid. The toroid presents a largely unshielded dark surface to thermal radiation. But below about $60 \mathrm{~K}$ the levitation force becomes limited by the field profile of the magnet[21], [22]. So even if $J_{c}$ increases, colder temperatures do not significantly affect the results. The field-independent $J_{c}$ reproduces the observations well, indicating that the actual field-dependence is weak in this field range.

The high stiffness and low hysteresis points to the bulks having a high $J_{c}[23]$. Damping could be increased, at the cost of reduced levitation force, by reducing $J_{c}$. This could be achieved, for example, by operating closer to $T_{c}$, thereby relaxing cooling requirements. Passive damping could also be improved by adding a thin copper eddy current damper[24].

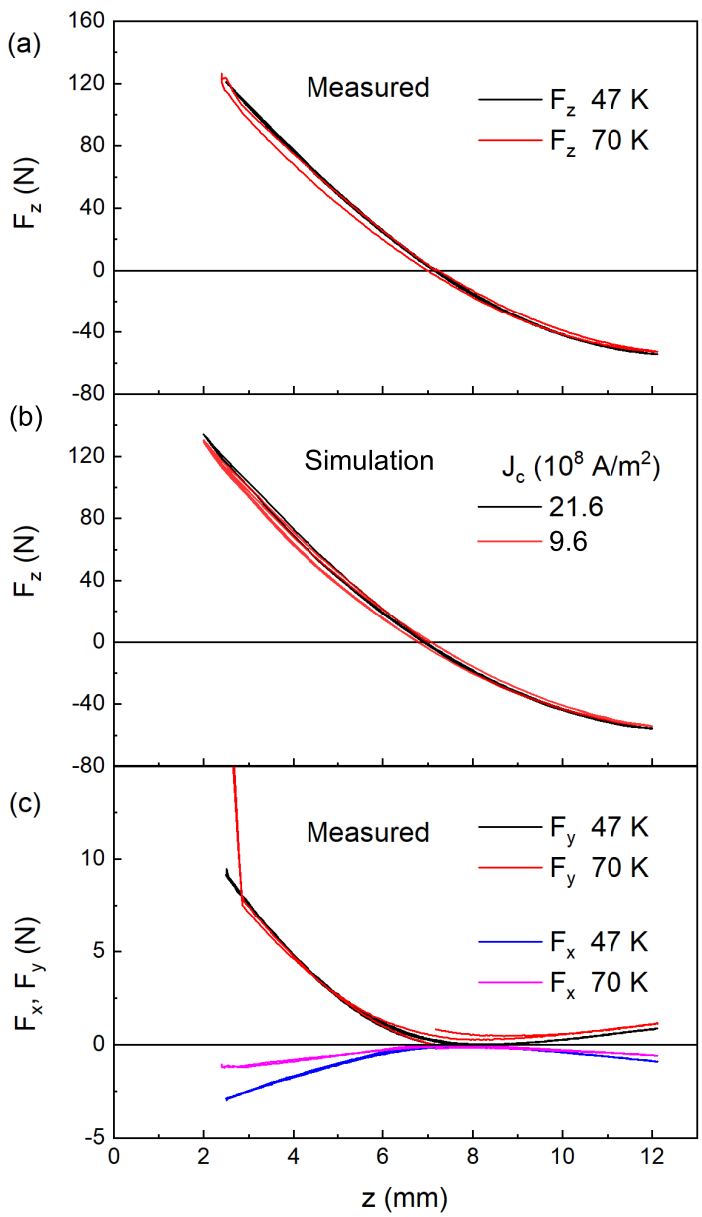

Fig. 7. Field-cooled levitation force as a function of levitation height. (a) Measured $z$-component at 47 and $70 \mathrm{~K}$. (b) Simulated $z$-component for two values of $J_{c}$. (c) Measured $x$ - and $y$-components at 47 and $70 \mathrm{~K}$.

An unexpected finding is that the forces from the puck and toroid are nonadditive. As an assembly, the centre puck plays no role and can be eliminated, reducing the amount of HTS used. Though not shown here, we have confirmed through simulations that this also holds under field-cooling conditions. Further simulations could be carried out to identify the optimal slope of the PM and toroid aperture.

So what advantages, if any, does the assembly and frustum PM configuration have over the traditional arrangement of a puck and cylindrical PM? Performing an equivalent comparison isn't as simple as replacing the assembly with a puck of equal outer diameter $(50 \mathrm{~mm})$. Firstly, we employ simulation which not subject to material variations between bulks. Secondly, we consider a much narrower minimum puckPM separation of $0.3 \mathrm{~mm}$, similar to the gap between the sides of the frustum PM and the inside face of the toroid when $z=2 \mathrm{~mm}$ (see Fig. 4(a)). Thirdly, we take a PM with radius $12.7 \mathrm{~mm}$, equal to the large radius of the frustum $\mathrm{PM}$, and a matching thickness of $12.7 \mathrm{~mm}$. These dimensions produce a similar FC force $(125 \mathrm{~N})$ at minimum separation as the frustum-assembly combination. Comparing the results in Fig. 9(a) reveals that the assembly has higher and more consistent vertical stiffness over most of the displacement 


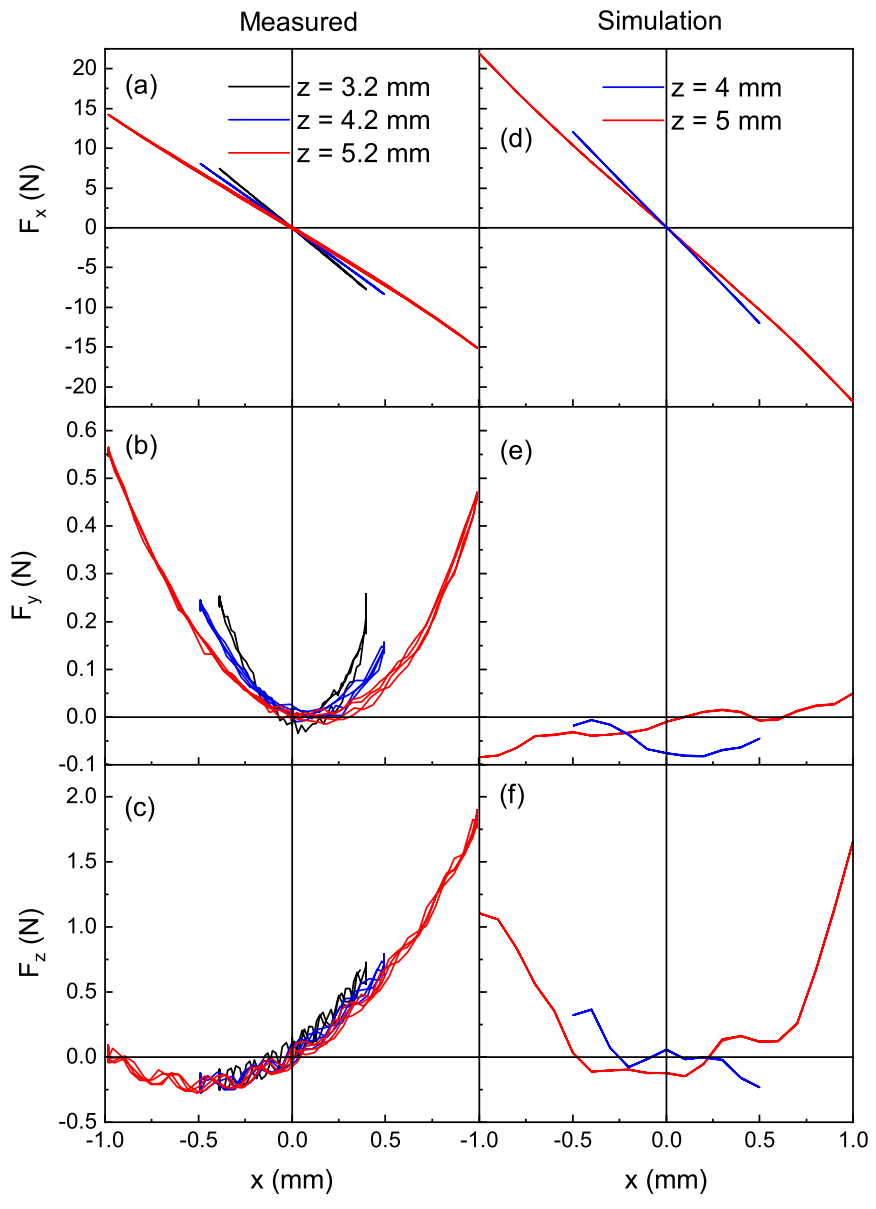

Fig. 8. (a) - (c) Measured force as a function of lateral displacement at $54 \mathrm{~K}$, after field-cooling with the PM at $z=3.2,4.2$ and $5.2 \mathrm{~mm}$. (d) - (f) Simulated force after field-cooling at $z=4 \mathrm{~mm}$ and $5 \mathrm{~mm}$, with $J_{c}=21.6 \times 10^{8} \mathrm{~A} / \mathrm{m}^{2}$.

range. Moreover, it generates superior restoring force for displacements above the field-cooling height of $7 \mathrm{~mm}$. Comparing lateral force performance is complicated by the fact that the minimum separation between the toroid and frustum PM is not constant. So we take the average separation, equal to the initial separation of $1.3 \mathrm{~mm}$ (when $[x, y, z]=[0,0,5] \mathrm{mm}$ ) as the fieldcooling height for the cylindrical PM. Figure 9(b) shows that for displacements less than $0.4 \mathrm{~mm}$ the assembly provides an improvement in stiffness of about 33 percent. But because the assembly has a more linear response, the performance advantage increases at higher displacements. At $x= \pm 1 \mathrm{~mm}$ the assembly produces just over double the lateral restoring force and around four times the stiffness. To provide similar performance, the cylindrical PM would need to be field-cooled at a much lower height of about $z=0.3 \mathrm{~mm}$. The obvious disadvantage of the assembly geometry is that the range of permissible lateral movement is restricted and depends on the vertical position of the PM.

In summary, we have shown that a conical frustum shaped permanent magnet levitated above a matched trapezoidal HTS toroid experiences superior stiffness and restoring forces compared to a conventional cylindrical PM and HTS puck arrangement. Our ongoing studies will evaluate the dynamic performance of the frustum-toroid configuration under high-
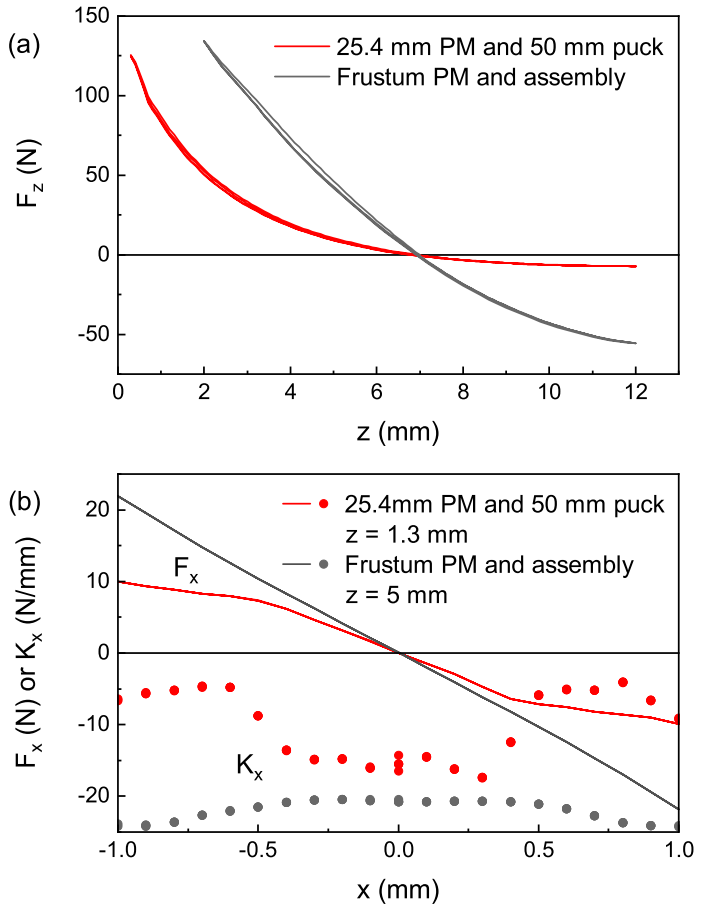

Fig. 9. (a) Simulated levitation force for a $25.4 \mathrm{~mm}$ diameter cylindrical PM above a $50 \mathrm{~mm}$ diameter HTS puck, after field-cooling at $z=7 \mathrm{~mm}$. The simulated values for the frustum PM with HTS assembly (toroid and puck) are shown for comparison. $J_{c}$ is $21.6 \times 10^{8} \mathrm{~A} / \mathrm{m}^{2}$. (b) Simulated lateral force (lines) and stiffness (circles) illustrating the superior performance of the assembly and frustum PM over the cylindrical PM and puck.

speed rotation of the PM to further assess its suitability for high-speed bearing applications.

\section{ACKNOWLEDGMENT}

The authors would like to thank Dr. Mark Ainslie (University of Cambridge) for helpful instruction on the $\mathrm{H}$ formulation method.

\section{REFERENCES}

[1] K. S. Haran, S. Kalsi, T. Arndt, H. Karmaker, R. Badcock, B. Buckley, T. Haugan, M. Izumi, D. Loder, J. W. Bray, P. Masson, and E. W. Stautner, "High power density superconducting rotating machines-development status and technology roadmap," Superconductor Science and Technology, vol. 30, no. 12, p. 123002, nov 2017. [Online]. Available: https://doi.org/10.1088/1361-6668/aa833e

[2] H. D. Kim, A. T. Perry, and P. J. Ansell, "A review of distributed electric propulsion concepts for air vehicle technology," in 2018 AIAA/IEEE Electric Aircraft Technologies Symposium (EATS), July 2018, pp. 1-21.

[3] S. Kalsi, K. Hamilton, R. Buckley, and R. Badcock, "Superconducting AC homolpolar machines for high-speed applications," Energies, vol. 12, no. 1, p. 86, 2019. [Online]. Available: https://doi.org/10.3390/en12010086

[4] S. S. Kalsi, J. Storey, K. Hamilton, and R. A. Badcock, Propulsion motor concepts for airplanes. [Online]. Available: https://arc.aiaa.org/doi/abs/10.2514/6.2019-4517

[5] J. R. Hull, "Superconducting bearings," Superconductor Science and Technology, vol. 13, no. 2, pp. R1-R15, jan 2000. [Online]. Available: https://doi.org/10.1088/0953-2048/13/2/201

[6] K. B. Ma, Y. V. Postrekhin, and W. K. Chu, "Superconductor and magnet levitation devices," Review of Scientific Instruments, vol. 74, no. 12, pp. 4989-5017, 2003. [Online]. Available: https://doi.org/10.1063/1.1622973 
[7] I. Valiente-Blanco, E. Diez-Jimenez, C. Cristache, M. A. AlvarezValenzuela, and J. L. Perez-Diaz, "Characterization and improvement of axial and radial stiffness of contactless thrust superconducting magnetic bearings," Tribology Letters, vol. 54, no. 3, pp. 213-220, Jun 2014. [Online]. Available: https://doi.org/10.1007/s11249-013-0204-0

[8] J. Ma, Y. Zhou, J. Li, B. Chen, and W. Fang, "The effect of cone additional permanent magnet thickness on magnetic field distribution and the levitation force of single domain GdBCO bulk superconductor," IOP Conference Series: Materials Science and Engineering, vol. 484, p. 012007, mar 2019. [Online]. Available: https://doi.org/10.1088/1757-899x/484/1/012007

[9] Can Superconductors, https://www.can-superconductors.com/. Kamenice, Czech Republic.

[10] SuperMagnetMan, https://supermagnetman.com/. Pelham, AL 35124, United States.

[11] COMSOL Multiphysics ${ }^{\circledR}$ v. 5.3a., http://www.comsol.com. COMSOL $\mathrm{AB}$, Stockholm, Sweden.

[12] L. Quéval, K. Liu, W. Yang, V. M. R. Zermeño, and G. Ma, "Superconducting magnetic bearings simulation using an H-formulation finite element model," Superconductor Science and Technology, vol. 31, no. 8, p. 084001, Jun 2018. [Online]. Available: https://doi.org/10.1088/1361-6668/aac55d

[13] Advanced Hall Sensors Ltd., https://www.ahsltd.com/p15a-hall-sensor. Manchester M17 1RW, United Kingdom.

[14] J. Duron, F. Grilli, B. Dutoit, and S. Stavrev, "Modelling the E-J relation of high- $T_{c}$ superconductors in an arbitrary current range," Physica C: Superconductivity, vol. 401, no. 1, pp. 231 - 235, 2004, proceedings of the International Cryogenic Materials Conference: Topical Conference on the VoltageCurrent Relation in Technical Superconductors. [Online]. Available: http://www.sciencedirect.com/science/article/pii/S0921453403015181

[15] A. Morandi, "2D electromagnetic modelling of superconductors," Superconductor Science and Technology, vol. 25, no. 10, p. 104003, sep 2012. [Online]. Available: https://doi.org/10.1088/09532048/25/10/104003

[16] M. D. Ainslie, D. Zhou, H. Fujishiro, K. Takahashi, Y.-H. Shi, and J. H. Durrell, "Flux jump-assisted pulsed field magnetisation of highjcbulk high-temperature superconductors," Superconductor Science and Technology, vol. 29, no. 12, p. 124004, oct 2016. [Online]. Available: https://doi.org/10.1088/0953-2048/29/12/124004

[17] M. D. Ainslie and H. Fujishiro, "Modelling of bulk superconductor magnetization," Superconductor Science and Technology, vol. 28, no. 5, p. 053002, mar 2015. [Online]. Available: https://doi.org/10.1088/09532048/28/5/053002

[18] V. Lahtinen, M. Lyly, A. Stenvall, and T. Tarhasaari, "Comparison of three eddy current formulations for superconductor hysteresis loss modelling," Superconductor Science and Technology, vol. 25, no. 11, p. 115001, sep 2012. [Online]. Available: https://doi.org/10.1088/09532048/25/11/115001

[19] P. Z. Chang, F. C. Moon, J. R. Hull, and T. M. Mulcahy, "Levitation force and magnetic stiffness in bulk high-temperature superconductors," Journal of Applied Physics, vol. 67, no. 9, pp. 4358-4360, 1990. [Online]. Available: https://doi.org/10.1063/1.344927

[20] J. R. Hull, "Effect of permanent-magnet irregularities in levitation force measurements," Superconductor Science and Technology, vol. 13, no. 6, pp. 854-856, may 2000. [Online]. Available: https://doi.org/10.1088/0953-2048/13/6/347

[21] J. Zhou, X.-Y. Zhang, and Y.-H. Zhou, "Temperature dependence of levitation force and its relaxation in a hts levitation system," Physica C: Superconductivity, vol. 470, no. 5, pp. 336 - 339, 2010. [Online]. Available: http://www.sciencedirect.com/science/article/pii/S0921453410001000

[22] A. Morandi, M. Fabbri, P. L. Ribani, A. Dennis, J. Durrell, Y. Shi, and D. Cardwell, "The measurement and modeling of the levitation force between single-grain ybco bulk superconductors and permanent magnets," IEEE Transactions on Applied Superconductivity, vol. 28, no. 5, pp. 1-10, Aug 2018.

[23] C. Navau and A. Sanchez, "Stiffness and energy losses in cylindrically symmetric superconductor levitating systems," Superconductor Science and Technology, vol. 15, no. 10, pp. 1445-1453, sep 2002. [Online]. Available: https://doi.org/10.1088/0953-2048/15/10/313

[24] Z.-F. Jiang and X.-F. Gou, "Eddy damping effect of additional conductors in superconducting levitation systems," Physica C: Superconductivity and its Applications, vol. 519, pp. $112-117,2015 . \quad$ [Online]. Available: http://www.sciencedirect.com/science/article/pii/S0921453415002658 